PlastOx 2007 (2009) 33-43

(C) EDP Sciences, 2009

DOI: $10.1051 /$ ptox/2009004

\title{
Outils de simulation à l'échelle atomique pour traiter la chimie et la plasticité
}

\author{
C. Domain
}

\author{
EDF R\&D, Département MMC, Les Renardières, 77250 Moret sur Loing, France \\ e-mail : christophe.domain@edf.fr
}

\begin{abstract}
Résumé. La plupart des mécanismes élémentaires de l'évolution de la microstructure et de la plasticité ont lieu à l'échelle atomique, et dépendent de la nature chimique des atomes présent dans le matériau. Par exemple, dans les matériaux à friction de réseau importante, la plasticité à basse température est contrôlée par le mouvement des dislocations vis et la structure de cœur des dislocations joue un rôle important. Les calculs atomiques permettent d'étudier précisément les propriétés de cœur, mais également les énergies de faute généralisées, les surfaces et les joints de grain qui peuvent apporter des données sur les mécanismes élémentaires.

Les résultats pouvant être obtenus seront illustrés dans le cas du Fe pour les cubiques centrés et le cas du Zr et du Ti seront présentés pour les hexagonaux et l'effet de l'hydrogène sera discuté. Les résultats de simulation de la mobilité de la dislocation vis dans le Fe par dynamique moléculaire seront comparés aux mesures expérimentales.
\end{abstract}

\section{INTRODUCTION}

Dans les métaux hexagonaux ou cubiques centrés, la plasticité à basse température est contrôlée par le mouvement des dislocations vis. Les propriétés élémentaires, telles que la structure de cœur ou les énergies de faute d'empilement, peuvent alors être corrélés au mouvement des dislocations et à la friction de réseau importante. Les calculs atomiques permettent d'étudier précisément les propriétés de cœur, et les calculs ab initio basés sur la théorie de la fonctionnelle de la densité sont les plus précis à ce jour pour aborder ces questions pour des composés purs, des alliages, incluant les effets d'éléments en solution (en substitution ou interstitiel). En complément des dislocations, les énergies de faute généralisées, les surfaces et les joints de grain particulier tels que les macles peuvent apporter des données sur les mécanismes élémentaires.

Il existe deux approches pour déterminer la structure de cœur d'une dislocation vis à partir de calculs $a b$ initio. La première est basée sur le modèle de Peierls Nabarro pour lequel l'étalement du cœur est obtenu en résolvant un ensemble d'équations utilisant l'énergie de faute d'empilement généralisée calculée par $a b$ initio. La structure de cour de différents métaux (e.g. Ni 3 Al (Schoeck 1999) [1], $\mathrm{Al}$ and Ag (Lu et al. 2003) [2] et semi-conducteurs (e.g. Si (Joos et al. 1994) [3]) ont ainsi été obtenue. La seconde consiste à calculer directement la structure de cœur relaxée, ce qui est plus facile pour les structures de cœur relativement peu étalées, non planaires et complexes, comme par exemple dans les métaux cubique centrés ou hexagonaux.

Le calcul direct de la structure de cœur de la dislocation vis $\langle 111\rangle$ a été calculé dans le Fe, le Mo et le Ta (Frederiksen et Jacobsen 2003, Ismail-Beigi and Arias 2000, Woodward et Rao 2002) [4-6]. Les calculs $a b$ initio prédisent une structure de cœur compacte et symétrique, différente de la structure en trèfle obtenue avec un grand nombre de potentiels empiriques utilisés en dynamique moléculaire.

Dans cet article, les résultats obtenus sont exposés dans le cas du Fe pour les cubiques centrés. Le cas du Zr et du Ti sont présentés pour les hexagonaux avec l'effet de l'hydrogène en solution solide. Le lien possible de la dynamique moléculaire validée par des calculs ab initio avec les modèles de cohésion est discuté pour le mouvement de la dislocation vis dans le Fe. 
Par ailleurs, l'étude de la plasticité d'alliage complexe sous sollicitations sévères comme l'irradiation requière une connaissance précise de la microstructure du matériau étudié. La compréhension et la prédiction de l'évolution des propriétés et de la microstructure nécessite de décrire les différents mécanismes physiques ayant lieu à différentes échelles d'espace et de temps : depuis l'interaction des défauts ponctuels et leur interaction avec la chimie du matériau à l'échelle atomique (et la formation des défauts ponctuels sous irradiation) à la diffusion des défauts ponctuels à moyen et long terme, jusqu'à l'évolution des propriétés plastiques et mécaniques du matériau. Une approche multi-échelle est alors mis en place permettant de chaîner et/ou coupler différentes techniques de modélisation suivant les mécanismes traités.

Quelques résultats relatifs à l'évolution de la microstructure d'oxyde et plus particulièrement la diffusion des cations seront également présentés après une présentation succinctes des différentes méthodes de simulation.

\section{MÉTHODES DE SIMULATION}

En fonction du mécanisme physique traité différentes méthodes de simulation sont utilisées (voir par exemple Raabe pour une revue de ces méthodes [7]). (i) La caractérisation des mécanismes élémentaires est obtenus par les calculs ab initio, description la plus précise à ce jour pour décrire la cohésion du matériau, et permettant de traiter relativement aisément plusieurs espèces atomiques simultanément. Les calculs ab initio sont très lourds et permettent de traiter au plus quelques centaines d'atome en faisant des calculs statiques sur des configurations relaxées. (ii) La dynamique moléculaire classique permet d'avoir la trajectoire d'un ensemble d'atome et elle décrit la cohésion du matériau à l'aide d'un potentiel d'interaction semi-empirique ajusté sur des propriétés élémentaires et/ou des résultats de calcul ab initio. La construction de potentiels pour des alliages multinaires est délicate et la plupart du temps des matériaux pures ou alliages binaires sont modélisés dans les métaux. Les simulations de dynamique moléculaire classique traitent des systèmes allant jusqu'à quelques millions d'atomes sur des durées de quelques nanosecondes. (iii) Les méthodes de Monte Carlo cinétique permettent de simuler la diffusion atomique. Elles permettent de simuler l'évolution de la microstructure sous irradiation ou par vieillissement thermique et fournissent la cinétique de formation d'amas de soluté, ségrégation et/ou amas de défauts ponctuels. (iv) Des méthodes telles que la dynamique d'amas permettent de décrire l'évolution de la microstructure sur de grande échelle de temps en considérant les concentrations et donc sans corrélations spatiales. (v) Enfin, les méthodes de champ de phase permette un traitement mésoscopique de la microstructure.

L'étude de la plasticité est basé principalement sur trois méthodes de simulation : (i) les calculs ab initio pour étudier précisément les propriétés de cœur des dislocations ; (ii) la dynamique moléculaire pour caractériser le mouvement d'une dislocation isolée ; (iii) la dynamique des dislocations permettant de traiter un ensemble de dislocations, pouvant présenter des précipités ou défauts d'irradiation par exemple.

Les calculs ab initio adaptés aux traitement des métaux sont basés sur la Théorie de la Fonctionnelle de la Densité (DFT) qui a été appliquée pour traiter avec succès différents problèmes en science des matériaux (Hafner 2000) [8]. La DFT permet d'obtenir la structure électronique dans l'état fondamental. Les principaux fondements sont : (i) l'énergie totale d'un système est une fonctionnelle unique de la densité électronique ; (ii) le minimum de cette fonctionnelle est l'état fondamentale du système ; (iii) l'hamiltonien totale (de tous les électrons, i.e. le problème à $\mathrm{N}$ corps) peut être formellement remplacé par un système d'hamiltoniens mono-électroniques (Hohenberg 1964, Kohn 1965, Parr et Yang 1989, Payne et al. 1992) [9-12].

Les calculs $a b$ initio présentés ici ont été réalisés avec le code VASP (Vienna Ab initio Simulation Package) (Kresse et al.) [13]. Dans ce code, les concepts de la DFT sont mis en ouvre pour résoudre l'hamiltonien de Kohn-Sham mono-électronique à l'aide d'algorithmes performants. Le développement des fonctions d'onde est effectué sur une base d'ondes planes avec des pseudo-potentiels ultra doux 
(de type Vanderbilt ou Projected Augmented Wave). Les termes d'échange et corrélation sont traités dans le cadre de l'approximation de la densité locale (LDA) avec les termes de gradient (GGA).

\section{MICROSTRUCTURE ET CHIMIE}

La stabilité des oxydes vis à vis de la mise en solution des cations les constituants avec le milieu extérieur est une propriété déterminant à la fois la composition du milieu et celle de l'oxyde. Par exemple, la stabilité du $\mathrm{Zn}$ vis à vis du Co ou du Ni dans les oxydes présent au niveau des tubes des générateurs de vapeurs peut avoir des conséquences sur la dosimétrie au voisinage du générateur de vapeur. Le Co et/ou le Ni peuvent s'activé au passage près du cœur et sont les éléments responsable de l'activité présente dans les tubes après re-déposition. En effet, il a été observé que l'addition de zinc dans le circuit primaire des centrales nucléaires conduit à une réduction des émissions radioactives au voisinage des générateurs de vapeur composés de tube en alliage 600. La couche d'oxyde est alors modélisée par un spinelle duplex composé d'une couche de chromite recouverte de cristallite de ferrite. Les énergies de substitution du Ni par du Co et du Zn ont alors été calculées par calcul ab initio [14].

La diffusion des solutés dans les oxydes de corrosion est une étape importante dans l'évolution de la composition des oxydes et l'échange chimique des cations avec le milieu aqueux. Les produits de corrosion formés en milieu aqueux sur des matériaux industriels sont complexes et sont composés d'un ensemble de phases. Afin de pouvoir les traiter par calculs atomiques (calculs ab initio) il est nécessaire de les représenter par différentes phases. Par exemple, les oxydes formés sur les bases nickels sont modélisés par une spinelle $\mathrm{NiCr}_{2} \mathrm{O}_{4}$ et de l'oxyde de chrome $\mathrm{Cr}_{2} \mathrm{O}_{3}$. Les calculs ab initio ont montré que les énergies de migration dans la spinelle sont beaucoup plus faible que dans l'oxyde de chrome, ce qui est cohérent avec le caractère protecteur de $\mathrm{Cr}_{2} \mathrm{O}_{3}$.

\section{PLASTICITÉ ET CHIMIE À L'ÉCHELLE ATOMIQUE}

\section{1 Énergie de faute et structure de cour des dislocations}

Pour calculer l'énergie d'excès correspondant à une faute d'empilement, on effectue une translation rigide de deux blocs de cristal situés de part et d'autre du plan de faute. Pour une translation donnée, l'énergie du système est minimisée en autorisant uniquement la relaxation des atomes dans la direction perpendiculaire au plan de glissement. L'énergie de faute est définie comme la différence d'énergie par unité de surface entre le cristal avec faute et le cristal parfait. La supercellule choisie présente des surfaces libres parallèles au plan de faute et une seule faute est introduite. Cette géométrie permet de considérer n'importe quel vecteur de translation et permet ainsi d'étudier l'ensemble de la surface $\gamma$ et donne accès à l'énergie de faute généralisée.

Pour étudier la structure de cœur des dislocation vis de vecteur de Burgers $\mathrm{b}=\langle\mathrm{a}\rangle$ dans le $\mathrm{Zr}$ (respectivement $\mathrm{b}=\mathrm{a} / 2\langle 111\rangle$ dans le $\mathrm{Fe}$ ), la ligne de dislocation est placée au centre d'une supercellule constituée d'un empilement de deux (respectivement trois) plans atomiques. Le champ de déplacement prédit par l'élasticité linéaire (b $\theta / 2 \pi)$ est appliqué à tous les atomes. Les atomes de la supercellule les plus éloignés de la ligne de dislocation constituent des surfaces, ils sont entourés de vide et sont fixes (Figure 1). De part les conditions périodiques aux limites, la ligne de dislocation est infinie. A partir de ces positions initiales, tous les atomes sauf ceux du bord sont relaxés par gradient conjugué.

La structure de cœur est représentée en utilisant les notations de Vitek (Vitek et al. 1970) [15].

\subsection{Contrainte critique de clivage}

La contrainte critique de clivage $\sigma_{\mathrm{c}}$ est un paramètre mécanique souvent utilisé pour définir un critère d'amorçage de fissures. Il s'agit d'une quantité qui dépend du chemin suivi pour séparer deux blocs de cristal [16], alors que le travail de séparation obtenu en intégrant la contrainte en fonction de l'écartement 


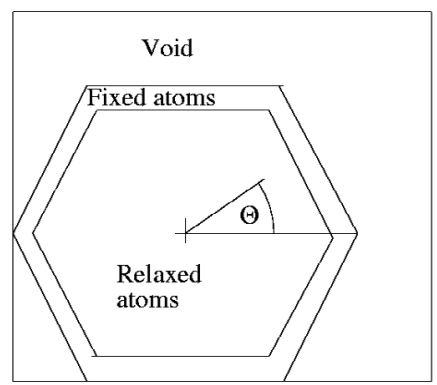

Figure 1. Schéma d'une supercellule pour simuler la structure de cœur d'une dislocation vis.

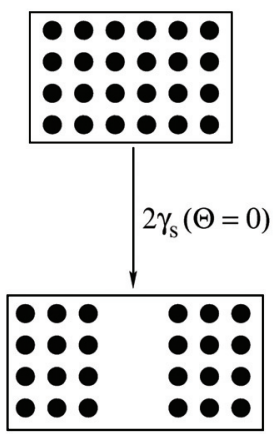

(a)

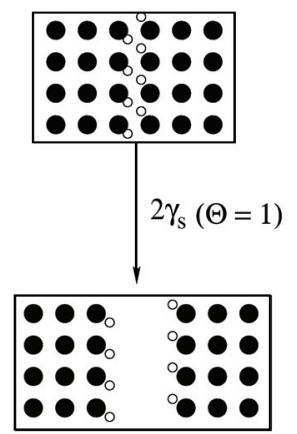

(b)

Figure 2. Supercellule utilisée pour le calcul de la rupture par écartement de deux blocs rigides contenant une espèces chimiques avec un taux de couverture $\theta$.

des blocs peut être une fonction d'état et à ce titre indépendante du chemin suivi. La procédure choisie pour déterminer sans ambiguïté $\sigma_{\mathrm{c}}$ consiste à enregistrer la variation de l'énergie totale du système en fonction de l'écartement $\delta$ de deux blocs rigides de cristal. La dérivée de cette courbe donne accès à la contrainte. En présence d'espèces chimiques dans le plan de séparation, telles que hydrogène ou iode, seul l'atome d'H ou d'I est autorisé à relaxer.

\section{3 Énergies de faute et surface gamma}

Les énergies de faute et énergies de faute généralisée dans le Zr et le Ti sont présentées ainsi que l'effet de l'hydrogène en solution solide.

\subsection{1 Énergies de faute}

Les énergies de faute basale et prismatique sont données dans la table 1 et sont qualitativement en bon accord avec les calculs de liaisons fortes de Legrand (1984) [17]. Les énergies de faute basale ( $\mathrm{Zr}$ : $200 \mathrm{~mJ} / \mathrm{m}^{2}$, Ti : $\left.291 \mathrm{~mJ} / \mathrm{m}^{2}\right)$ sont plus élevées que les valeurs pour le plan prismatique $\left(\mathrm{Zr}: 145 \mathrm{~mJ} / \mathrm{m}^{2}\right.$, Ti : $174 \mathrm{~mJ} / \mathrm{m}^{2}$ ). La prise en compte des relaxations atomiques est indispensable pour obtenir une énergie de faute prismatique $\gamma_{\text {pris }}$ plus faible que la faute basale $\gamma_{\text {bas }}$ pour laquelle la relaxation est faible (la relaxation ne représente que 15\%).

Les résultats $a b$ initio sont en accord avec la seule mesure expérimentale obtenue pour le $\mathrm{Ti}$ : $150 \mathrm{~mJ} / \mathrm{m}^{2}$ (De Crecy et al. 1983). En supposant un cœur de dislocation dissocié en deux partielles, les résultats précédents prédisent un étalement prismatique préférentiel. 
Tableau 1. Énergie de faute de $\mathrm{Zr}$ et $\mathrm{Ti}$, constantes élastiques de cisaillement et rapport de Legrand (les valeurs entre parenthèses sont non relaxées).

\begin{tabular}{|c|c|c|c|c|c|}
\hline & $\gamma_{\text {bas }}\left(\mathrm{mJ} / \mathrm{m}^{2}\right)$ & $\gamma_{\text {prism }}\left(\mathrm{mJ} / \mathrm{m}^{2}\right)$ & $\mathrm{C}_{44}(\mathrm{GPa})$ & $\mathrm{C}_{66}(\mathrm{GPa})$ & $\mathrm{R}$ \\
\hline $\mathrm{Zr}$ & & & & & \\
\hline GGA & $200(237)$ & $145(455)$ & 29 & 39 & 1.9 \\
\hline TB (Legrand) [17] & 340 & 150 & 42 & 42 & 2.3 \\
\hline exp. & - & - & 36.3 & 44 & Prismatique \\
\hline Ti & & & & & \\
\hline GGA & 291 & 174 & 43 & 45 & 1.8 \\
\hline TB (Legrand) [17] & 290 & 110 & 45 & 43 & 2.5 \\
\hline exp. & - & 150 & 46.7 & 35 & Prismatique \\
\hline
\end{tabular}
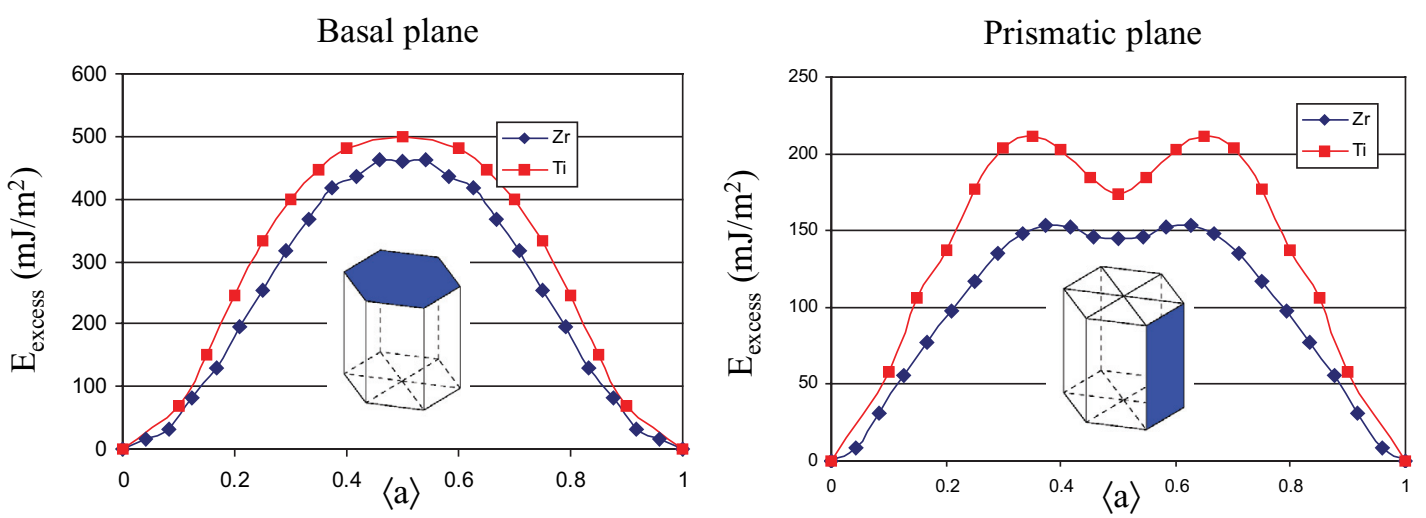

Figure 3. Profil d'énergie d'excès $\left(e n \mathrm{~mJ} / \mathrm{m}^{2}\right.$ ) le long du vecteur de Burgers $\langle\mathrm{a}\rangle$ dans le plan basal et prismatique pour le $\mathrm{Zr}$ et le Ti.

Legrand (1984) [17] a montré que les propriétés de glissement dans les matériaux hexagonaux sont décrites par le paramètre $R=C_{66} \gamma_{\text {bas }} / C_{44} \gamma_{\text {prism }}$. R est le rapport des distances de dissociation d'une dislocation dissociée dans le plan prismatique par rapport à celle dissociée dans le plan basal. $\mathrm{R}<1$ correspond à un étalement basal et $\mathrm{R}>1$ à un étalement prismatique. Les valeurs de $R$ obtenues pour le Ti et le $\mathrm{Zr}$ conduisent à un étalement prismatique préférentiel de la dislocation vis, cohérent avec le glissement prismatique observé expérimentalement (Akhtar et Teghtsoonian 1971) [18].

Le profil d'énergie d'excès le long du vecteur de Burgers $\langle a\rangle$ dans le plan basal et le plan prismatique pour le $\mathrm{Zr}$ et le Ti (Figure 3) montre que la faute prismatique à mi-chemin correspond à un minimum local qui est plus marqué pour Ti par rapport à Zr. Dans le plan basal, le profil le long du vecteur de burgers ne comprend pas la faute basale stable.

La figure 4 représente l'ensemble des surfaces $\gamma$ basal et prismatique dans le cas du Zr. Dans le plan prismatique le chemin de plus basse énergie est le long du vecteur de Burgers $\langle a\rangle$, passant ainsi par la faute basale à mi-distance. Dans le plan basal, le chemin de plus basse énergie suit un chemin en zigzag (suivant les directions $\langle-1 \quad 100\rangle$ et $\left\langle\begin{array}{llll}1 & 0 & -1 & 0\end{array}\right)$ ) contenant également la faute basale stable.

\subsubsection{Effet de l'hydrogène sur les énergies de faute}

L'étude par calculs $a b$ initio de l'influence de l'hydrogène sur la mobilité des dislocations est difficilement traitable directement. L'effet de l'hydrogène a donc été étudié de manière indirecte en plaçant de l'hydrogène dans le plan de faute pour différents taux de couverture (Domain et al. 2004) [19]. Pour le $\mathrm{Zr}$ et le $\mathrm{Ti}$, la présence d'hydrogène abaisse significativement les énergies de faute basale et prismatique, d'autant plus que le taux de couverture est élevé (cf. Table 2). Dans certains cas, l'énergie de 


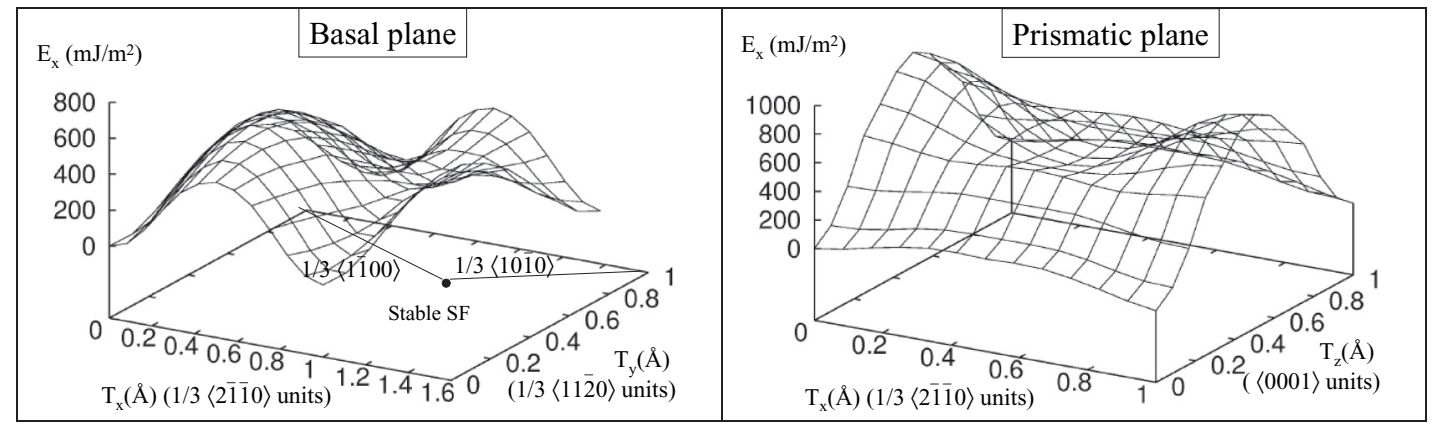

Figure 4. Profil d'énergie d'excès généralisée (en $\mathrm{mJ} / \mathrm{m}^{2}$ ) dans le plan basal et prismatique pour le $\mathrm{Zr}$.

Tableau 2. Effet de l'hydrogène (taux de couverture $\Theta$ ) sur les énergies de faute basale et prismatique dans le $\mathrm{Zr}$ et le $\operatorname{Ti}\left(\mathrm{en} \mathrm{mJ} / \mathrm{m}^{2}\right)$.

\begin{tabular}{cc|rrrrr}
\hline \multirow{2}{*}{$\Theta$} & \multicolumn{2}{c}{ plan basal } & & \multicolumn{2}{c}{ plan prismatique } \\
\cline { 2 - 3 } \cline { 5 - 6 } & $\mathrm{Zr}$ & $\mathrm{Ti}$ & & $\mathrm{Zr}$ & $\mathrm{Ti}$ \\
\hline 0 & 200 & 291 & & 145 & 174 \\
0.25 & 80 & 102 & & 73 & 72 \\
0.5 & -60 & -105 & & 67 & 66 \\
1 & -14 & -54 & & -90 & -190 \\
\hline
\end{tabular}

faute peut devenir négative ; ce résultat s'explique par la formation d'hydrures avec une grande énergie de formation (Domain 2002, Domain et al. 2004) [19, 20]. Le cœur de la dislocation vis pouvant être $\mathrm{vu}$ avec un ruban de faute prismatique, l'hydrogène pourrait potentiellement ségrégé dans le cœur de la dislocation vis ; l'effet est purement chimique dans la mesure où il n'y a pas d'interaction élastique entre la dislocation vis et l'hydrogène. Par ailleurs, en réduisant l'énergie de faute prismatique, l'hydrogène devrait accroître le glissement planaire.

\subsection{Structure de cœur des dislocations vis}

\subsubsection{Dislocation vis $\langle a\rangle$ dans le Zr et Ti}

En partant de la structure parfaite, les structures de cœur relaxées sont représentées sur les Figures 5 et 6 . Cette structure est obtenue uniquement si la relaxation des atomes est autorisée à la fois le long et perpendiculairement à la ligne de dislocation. La seule relaxation parallèle à la ligne est très faible et ne modifie pas sensiblement le structure de cœur donnée par le champ de déformation élastique. La relaxation des atomes dans le plan perpendiculaire à la ligne, correspondant à la composante coin, est donc crucial (Domain et Legris 2003) [21].

Ce comportement est quasi indépendant de la taille du système ; des calculs réalisés avec 61 atomes au lieu des 127 atomes présentés conduisent au même résultat (cependant avec un système plus petit Ferrer (2000) [22] avait un étalement moins important). L'étalement prismatique, de l'ordre de 3c, est déjà quasi-convergé avec une supercellule de 61 atomes. Les étalements secondaires observés, plus faible que l'étalement prismatique semblent assez étalées.

La structure de cœur obtenues par $a b$ initio est très différente des structures de cœur obtenues avec les potentiels empiriques pour lesquels un étalement basal est le plus favorable, en accord avec la faible énergie de faute basale prédite (par rapport à l'énergie de faute prismatique élevée), mais en désaccord avec les calculs ab initio et le glissement prismatique observé expérimentalement [23]. 


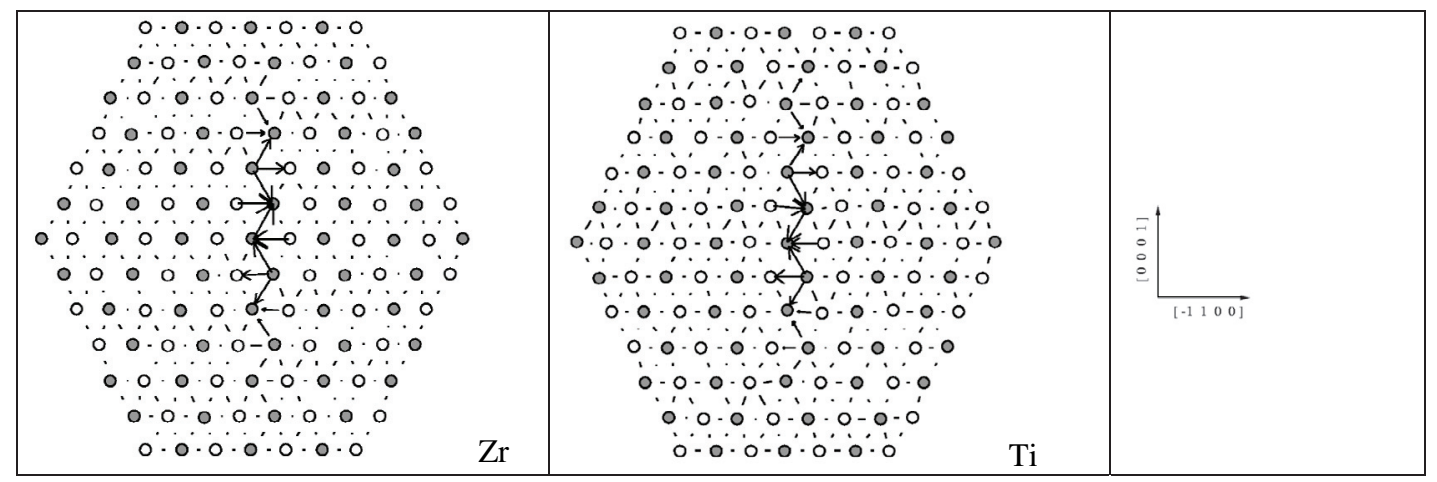

Figure 5. Structure de cœur de la dislocation vis dans le $\mathrm{Zr}$ et le Ti. Supercellule à 127 atomes.

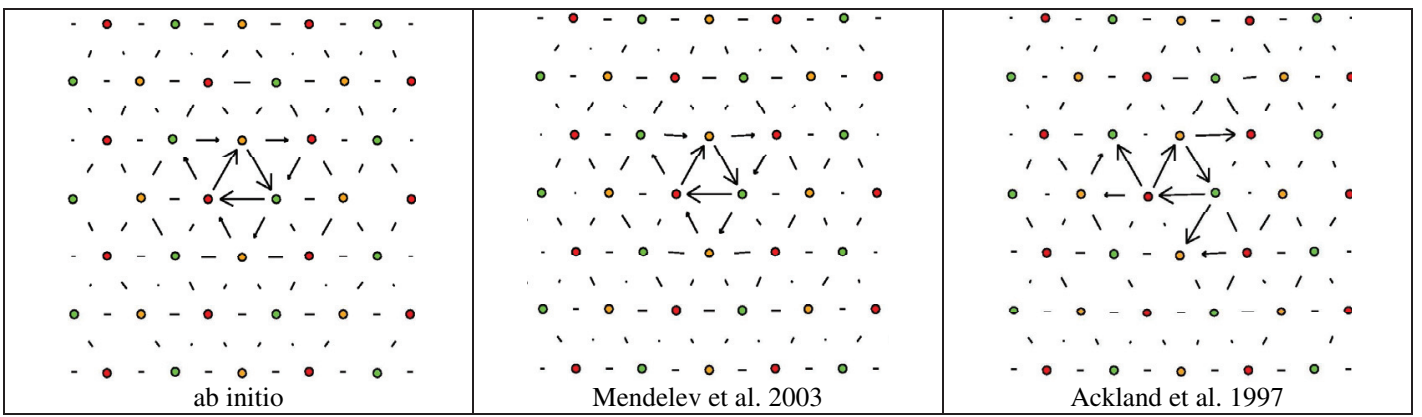

Figure 6. Structure de cœur de la dislocation vis a/2 $\langle 111\rangle$ dans le Fe obtenue par ab initio et avec différents potentiels empiriques.

\subsubsection{Dislocation vis a/2〈111〉 dans le Fe}

La structure de cœur relaxée de la dislocation vis a/2 $\langle 111\rangle$ dans le Fe obtenue par ab initio est représentée sur la Figure 6(a). La structure de cœur est similaire à celle de Frederiksen et Jacobsen (2003) [4] calculée en introduisant un dipôle de dislocation avec des conditions aux limites périodiques volumiques. Un cœur compact est obtenu cohérent avec les autres calculs ab initio réalisés dans les métaux cc (Ta, Mo) en utilisant des conditions aux limites similaires aux nôtres (Ismail-Beigi et al. 2000) [5] ou plus sophistiquées avec des fonctions de Green (Woodward et Rao 2002) [6].

Parmi les potentiels empiriques, utilisé en dynamique moléculaire, modélisant le Fe, seul le potentiel récemment développé par Mendelev et al. (2003) [24] conduit à une structure de cœur similaire. Dans l'ajustement de ce potentiel les énergies de formation des interstitiels obtenues par ab initio avait été prise en compte. Les autres potentiels de Fe (tels que ceux développés par Ackland et al. 1997 [25] ou Ludwig et al. 1998 [26]) prédisent une structure en trèfle qui n'est pas compatible avec le glissement préférentiel dans le plan (110) observé expérimentalement ; la dislocation glissant plutôt dans les plans (112). Par ailleurs, les contraintes de Peierls obtenues sont largement surestimées (Harry and Bacon 2002 [27], Marian et al. 2004 [28]).

\subsection{Mobilité de la dislocation vis dans le Fe}

Le potentiel de Mendelev et al. (2003) [24], pour lequel les propriétés de cœur de la dislocation vis dans le Fe ont été validées sur les calculs ab initio, a été utilisé pour caractériser le mouvement de la dislocation vis à une température donnée par dynamique moléculaire par cisaillement d'un cristal à 


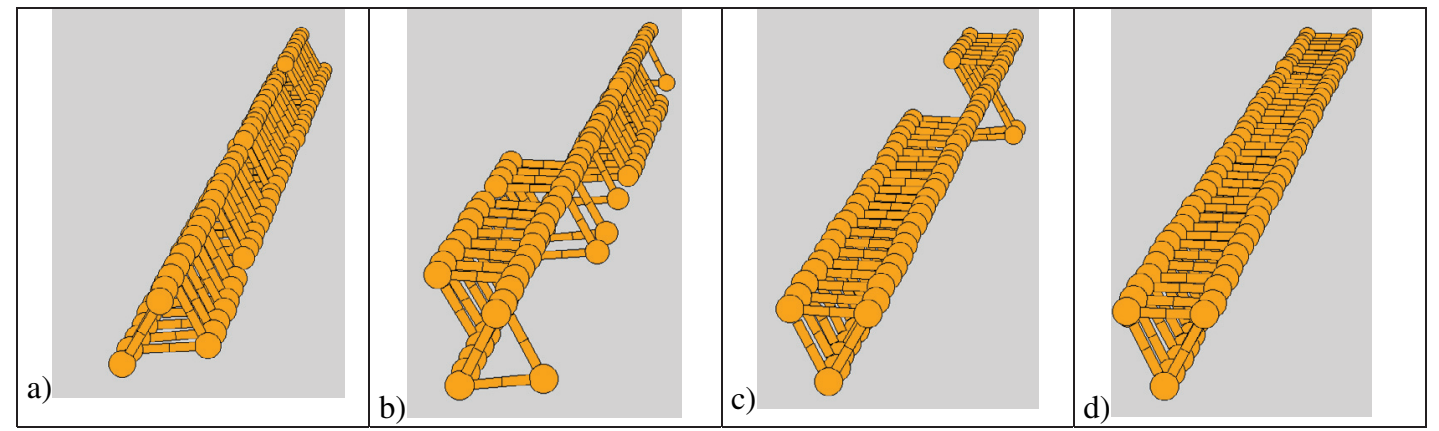

Figure 7. Nucleation et propagation d'un double-décrochement à $75 \mathrm{~K}$ par dynamique moléculaire d'une position "soft" a) à "hard" d).

vitesse constante (voir Domain et Monnet 2005 [29] pour plus de détails sur les simulations et l'analyse des résultats).

Avec ce potentiel la dislocation vis glisse dans le plan (110) en accord avec les observations expérimentales (Kubin 1976) [30]. Le mouvement s'effectue par nucléation et propagation d'un doubledécrochement (Figure 7). Ainsi par une succession de double-décrochements la dislocation glisse dans le plan (110) passant alternativement d'une position «soft » à une position « hard ».

Tous les modèles théoriques (e.g. Smidt 1969 [31], Louchet et Kubin 1979 [32]) supposent que la probabilité de nucléation d'un double-décrochement est proportionnelle au nombre de site de nucléation le long de la ligne de dislocation (i.e. la longueur de ligne $L$ ). Ils supposent également que l'effet principal de la contrainte est de réduire l'énergie d'activation $\triangle \mathrm{G}$. Un développement en série de Taylor en fonction de la contrainte donne :

$$
\Delta G=B-V \tau_{e f f},
$$

où $B$ est une constante et $V$ le volume d'activation ; ce qui conduit pour nos simulations à la relation suivante entre la vitesse moyenne $v$ et la contrainte critique $\tau_{\mathrm{c}}$ :

$$
v=A L \exp \left(-\frac{B-V \tau_{c}}{k T}\right)=A L\left\langle\exp \left(-\frac{B-V \tau_{e f f}}{k T}\right)\right\rangle,
$$

où $A$ est une constante indépendante de la contrainte, et $\langle\ldots\rangle$ représente la valeur moyenne. La longueur de ligne $L$ est constante dans la mesure où un seul double-décrochement se forme à la fois dans les simulations de dynamique moléculaire. La contrainte critique effective est alors donnée par :

$$
\tau_{c}=\frac{k T}{V} \ln \left\langle\exp \left(\frac{V \tau_{e f f}}{k T}\right)\right\rangle .
$$

Le volume d'activation, constant dans nos simulations, a été pris de l'ordre de $20 b^{3}$ (obtenus à partir d'expériences sur des mono-cristaux, Spitzig 1973 [33]).

L'évolution de la contrainte effective en fonction de la température montre une dépendance importante avec la température (Figure 8). La différence entre les données expérimentales et les données de simulations brutes est due au caractère dynamique et probabiliste du mécanisme de doubledécrochement.

En effet, les longueurs et vitesses de la dislocation vis sont très différentes dans les simulations et dans les expériences et ces différences doivent être considérées explicitement dans la comparaison. Il a été montré (Spitzig et Keh 1970 [34]) que la contribution de l'entropie à l'énergie de Gibbs est inférieure à $5 \%$ dans le cas du fer et est donc négligeable. Ainsi l'enthalpie obtenue par simulation est reliée à celle obtenue expérimentalement par la relation suivante faisant intervenir les vitesses et 


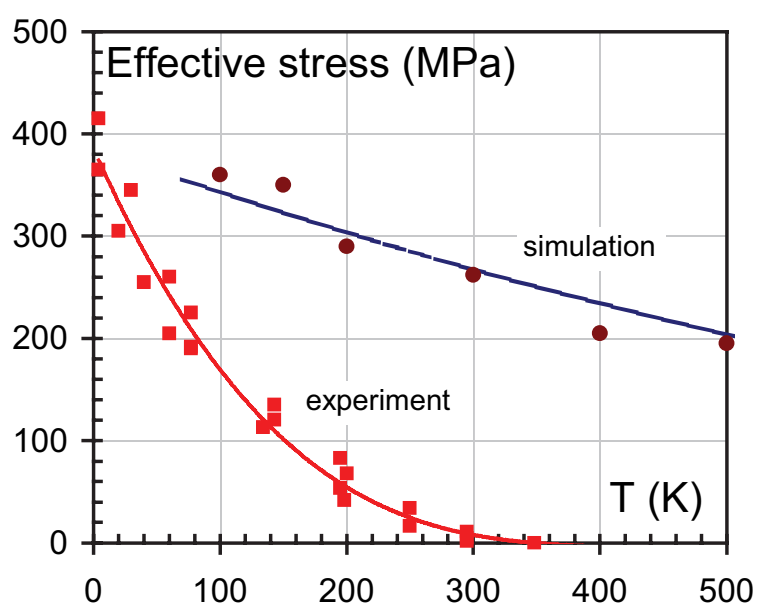

Figure 8. Évolution de la contrainte critique effective dans le plan (110) en fonction de la température à taux de déformation imposé. Les carré représentent les mesures expérimentales (exp-CES) et les cercles les résultats de dynamique moléculaire (MD-CES).

longueurs de dislocation :

$$
\Delta H_{\text {sim }}=\Delta H_{\exp }+k T \ln \frac{v_{\exp } L_{s i m}}{v_{s i m} L_{\exp }} .
$$

En raison des différences de conditions, le second terme est toujours négatif et est de l'ordre de $21 \mathrm{kT}$ dans le cas de nos simulations. C'est pourquoi les contraintes critiques effectives obtenues par simulation sont légèrement supérieures à celles mesurées expérimentalement. L'enthalpie d'activation mesurée est proportionnelle à la température et est de l'ordre de $\Delta \mathrm{H}_{\text {exp }}=25 k T$ dans le Fe. Dans nos simulations, on en déduit $\Delta \mathrm{H}_{\text {sim }}=4 k T$, ce qui est en accord avec l'effet moins prononcé de la température sur les résultats de dynamique moléculaire.

La différence d'enthalpie entre simulation et expérience est proportionnelle à la température. Ainsi une simulation réalisée à une température $T$ est équivalente à une expérience réalisée à $(4 k T / 25 k)=$ $0.16 T$; et une contrainte mesurée à une température $T$ est équivalente à une simulation à $1 / 0.16 T=$ 6.25 T. La courbe bleue de la figure 8 obtenue à partir des mesures expérimentales montrent l'excellent accord avec les résultats de dynamique moléculaire.

En résumé, l'utilisation d'un potentiel empirique, reproduisant une structure de cœur de la dislocation vis identique à celle prédite par les calculs $a b$ initio, conduit à des résultats en accord avec la théorie du double-décrochement pour le mouvement des dislocations et à des contraintes effectives comparables à celles mesurées expérimentalement sur des monocristaux de fer ultra-pur.

\subsection{Fracture et contrainte de clivage}

Dans l'analyse de la rupture fragile, la propagation de la fissure repose sur le concept de clivage en terme de travail et de contrainte critique. Le travail par unité de surface s'identifie simplement avec l'énergie de surface dans la mesure où le processus est lent et que l'équilibre chimique a lieu. En revanche la contrainte critique de clivage, déduite de la dérivée de la courbe énergie-déplacement, dépend fortement du chemin suivi par le système dans l'espace des phases. Ainsi, Hirth et Rice (1980) [16] ont défini différents processus de décohésion conduisant à différentes définitions de $\sigma_{\text {crit }}$.

Les énergies de surface avec et sans hydrogène sont données dans la table 3. La présence d'hydrogène conduit à une réduction significative de l'énergie de surface. L'effet plus important obtenu 
Tableau 3. Effet de H sur l'énergie de surface et la contrainte critique de clivage dans le Zr pour les plans basal et prismatique.

\begin{tabular}{|c|c|c|}
\hline & Basal & Prismatique \\
\hline $\mathrm{E}_{\text {surf }}\left(\mathrm{mJ} / \mathrm{m}^{2}\right) \theta=0$ & 1560 & 1644 \\
\hline $\mathrm{E}_{\text {surf }}\left(\mathrm{mJ} / \mathrm{m}^{2}\right) \theta=1$ & 764 & 1187 \\
\hline$\sigma_{\mathrm{c}}(\mathrm{GPa}) \theta=0$ & 16.2 & 15.7 \\
\hline$\sigma_{\mathrm{c}}(\mathrm{GPa}) \theta=1$ & 14.6 & 14.6 \\
\hline
\end{tabular}
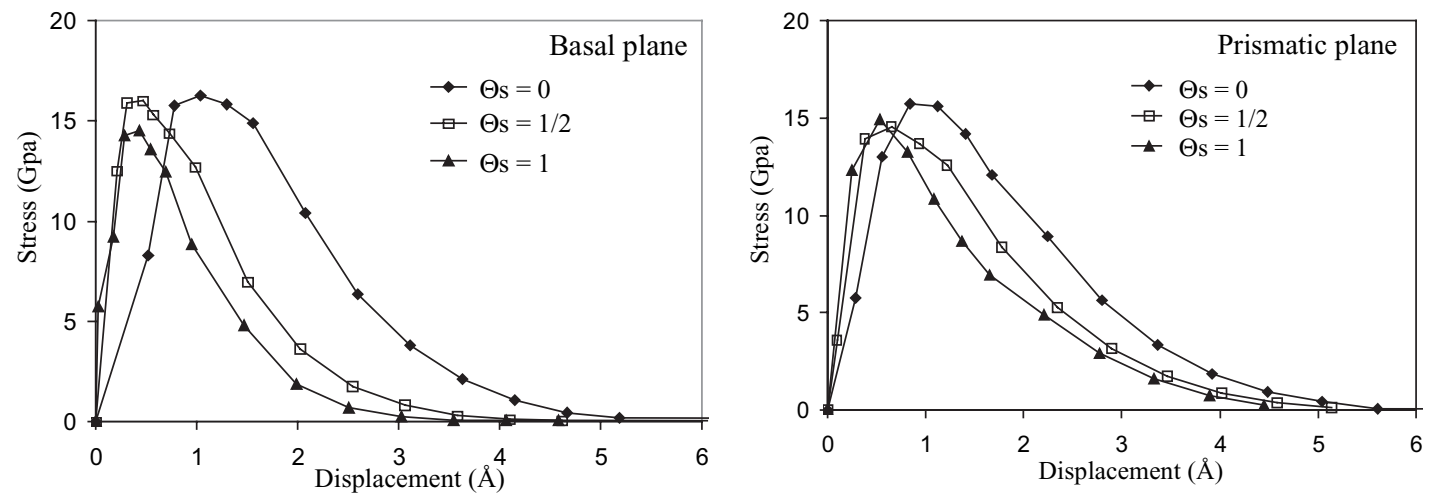

Figure 9. Effet de $\mathrm{H}$ sur la courbe contrainte-déplacement dans le $\mathrm{Zr}$ pour le clivage du plan basal et prismatique.

pour le plan basal par rapport au plan prismatique est vraisemblablement dû à la densité plus faible du plan prismatique pour un même taux de couverture $\theta$ en hydrogène [19].

L'effet de $\mathrm{H}$ sur la contrainte critique de clivage a été obtenu en simulant des tests de traction sur un monocristal dans les directions perpendiculaires aux plans basal et prismatique. La courbe de contraintedéformation (Figure 9) montre que la contrainte critique de clivage telle que nous l'avons définie est peu modifiée par la présence d'hydrogène, alors que l'effet sur l'énergie de surface était important.

Ce résultat est en désaccord avec la relation habituelle reliant énergie de surface et contrainte critique de clivage (critère de nucléation de fissure) :

$$
\sigma_{\text {crit }}=\sqrt{\frac{\gamma_{s} E}{a}}
$$

où $E$ est le module d'Young et $a$ la distance inter-planaire. Le système $\mathrm{Zr}$-H semble donc être un cas pour lequel la nucléation et la propagation de la fissure ne sont pas nécessairement liées simplement.

\section{CONCLUSIONS}

Les calculs $a b$ initio permettent une étude directe de la structure de cœur des dislocations lorsque celles-ci ne sont pas dissociées, comme dans les métaux hexagonaux ou cubique centrés. Ces calculs permettent de valider les potentiels empiriques utilisés en dynamique moléculaire pour les corps purs. Les effets des éléments chimiques en solution peuvent être abordé facilement dans la mesure où les interactions d'ordre chimique sont explicitement prises en compte.

Les calculs $a b$ initio sont précis mais en contre partie ne permettent de traiter qu'une centaine d'atomes. Les conditions aux limites ne sont donc pas toujours facile à considérer et l'étude de grandeurs élémentaires est plus aisée de ce point de vue. Les défauts plans tels que les fautes d'empilement et les fautes généralisées ou les surfaces permettent facilement d'aborder l'effet de la composition chimique avec une grande précision sur les énergies.

Les grandeurs calculées par $a b$ initio permettent d'alimenter des modèles analytiques en terme de données d'entrée ou bien d'effectuer des simulations aux échelles supérieures (en dynamique 
moléculaire par exemple) pour lesquelles les résultats sont plus facilement comparable à des grandeurs macroscopiques qui peuvent être confrontées aux résultats expérimentaux.

\section{Remerciements}

Ces travaux ont été réalisé dans le cadre du réseau européen ITEM (FIR1-CT-2001-20163) et des projets européens SIRENA (SIR1-CT-2001-20137) et PERFECT (FI6O-CT-2003-508840).

\section{Références}

[1] Schoeck G., Phil. Mag. Lett. 79 (1999) 849.

[2] Lu G., Bulatov V.V. and Kioussis N., Phil. Mag. 83 (2003) 3539.

[3] Joos B., Ren Q. and Duesbery M.S., Phys. Rev. B 50 (1994) 5890.

[4] Frederiksen S.L. and Jacobsen K.W., Phil. Mag. A 83 (2003) 365.

[5] Ismail-Beigi S. and Arias T.A., Phys. Rev. Lett. 84 (2000) 1499.

[6] Woodward C. and Rao S.I., Phys. Rev. Lett. 88 (2002) 216402.

[7] Raabe D., Computational materials science, Wiley-Vch (1998).

[8] Hafner J., Acta Mater. 48 (2000) 71.

[9] Hohenberg P. and Kohn W., Phys. Rev. 136 (1964) B864.

[10] Kohn W. and Sham L., Phys. Rev. 140 (1965) A1133.

[11] Parr R.G. and Yang W., Density functional theory of atoms and molecules, Oxford University Press (1989).

[12] Payne M.C., Teter M.P., Allan D.C., Arias A. and Joannopoulos J.D., Rev. Mod. Phys. 64 (1992) 1045.

[13] Kresse G. and Hafner J., Phys. Rev. B 47 (1993) R558; ibid. 49 (1994) 14251; Kresse G. and Furthmuller J., Phys. Rev. B 54 (1996) 11169; Kresse G. and Furthmuller J., Comput. Mat. Sci. 6 15 (1996) [http://cms.mpi.univie.ac.at/vasp/].

[14] Domain C., Guinard L., Doukhan J.C. and Legris A., Proceedings of the Conference « Water Chemistry of Nuclear Reactor Systems 8 », BNES, Bournemouth, UK, 2000

[15] Vitek V., Perrin R.C. and Bowen D.K., Philos. Mag. 21 (1970) 1049.

[16] Hirth J.P. and Rice J.R., Metall. Trans. A 11 (1980) 1501.

[17] Legrand B., Thèse, Orsay University, 1984.

[18] Akhtar A. and Teghtsoonian A., Acta Metall. 19 (1971) 655.

[19] Domain C., Legris A. and Besson R., Acta Mater. 52 (2004) 1495.

[20] Domain C., Thèse, Université de Lille 1 et EdF R\&D (2002).

[21] Domain C. and Legris A., Proceedings IUTAM conference, Osaka, 2003.

[22] Ferrer F., PhD Thèse, Ecole Polytechnique (2000).

[23] De Crecy A., Bourret A., Naka S. and Lasalmonie A., Phil. Mag. A 47 (1983) 245.

[24] Mendelev M.I., Han S.W., Srolovitz D.J., Ackland G.J., Sun D.Y. and Asta M., Phil. Mag. 83 (2003) 3977.

[25] Ackland G.J., Bacon D.J., Calder A.F. and Harry T., Phil. Mag. A 75 (1997) 713.

[26] Ludwig M. Farkas D., Predaza D. and Schmauder S., Modell. Simul. Mater. Sci. Eng. 6 (1998) 19.

[27] Harry T. and Bacon D.J., Acta Mater. 50 (2002) 195.

[28] Marian J., Cai W. and Bulatov V.V., Nat. Mater. 3 (2004) 158.

[29] Domain C. and Monnet G., Phys. Rev. Lett. 95 (2005) 215506.

[30] Kubin L.P., Rev. Deform. Behavior Mater. 1 (1976) 243.

[31] Smidt F.A., Acta Metall. 17 (1969) 381.

[32] Louchet F. and Kubin L.P., Phys. Stat. Solidi A 56 (1979) 169.

[33] Spitzig W.A., Mater. Sci. Eng. 12 (1973) 191.

[34] Spitzig W.A. and Keh A.S., Acta Metall. 18 (1970) 1021. 
